\title{
Éditorial
}

\section{Les femmes à l'honneur}

Ce n'est pas l'habitude, mais dans un premier temps, je parlerai dans cet éditorial d'un livre ; «Leçons de Marie Curie (Physique élémentaire pour les enfants de nos amis), recueillies par Isabelle Chavannes en 1907» (EDP Sciences, 2003, ISBN : 2-86883-635-6). C'est un document exceptionnel que publie EDP Sciences. Le manuscrit écrit de la main d'une des élèves de Marie Curie, Isabelle Chavannes, a été retrouvé par miracle dans une cave. Quelle chance !

À l'initiative d'un groupe d'amis, une expérience originale a été menée en 1907 et 1908 ; les parents se répartirent alors les principales matières à enseigner, les élèves étaient leurs enfants âgés alors d'une dizaine d'années. Certes ces enfants étaient de filiation illustre, Hadamard, Curie, Langevin, Perrin ou Chavannes, mais ceci ne retire rien à la fraîcheur de l'ouvrage où l'on voit comment Marie Curie a imaginé des expériences simples pour distinguer le vide de l'air, ce que cet air pèse sur nos épaules, comment l'eau arrive au robinet, etc.

Ce livre n'a pas pris une ride, il me rappelle un professeur de physique que j'ai eu en propédeutique, Maurice Françon : il nous expliquait la physique en nous faisant des expériences simples. C'était passionnant et son cours s'appelait tout simplement «Physique expérimentale ».

Bref un livre que je conseille à tous les enseignants ; il va, je l'espère, rendre jaloux notre président émérite, Daniel Blanc, qui anime la nouvelle rubrique, «Un peu d'histoire». Vous découvrez pour la première fois cette rubrique dans ce numéro. D'autres articles suivront, je l'espère.

Dans un second temps, je parlerai d'une seconde femme que nous connaissons tous, notre ancienne présidente Annie Sugier. Pourquoi ? D'abord elle est la première femme à prendre la tête d'un comité de la Commission internationale de protection radiologique (le 4: Application des recommandations de la commission), un exploit dans ce monde un peu machiste. Seule, avant elle, Angelina Guskova (le médecin de Tchernobyl) avait franchi les murs de cette citadelle en occupant de 1989 à 1993 un siège à la Main Commission, mais elle ne présidait pas de comité.

Plus important encore, nommée à la tête du comité 4, elle est chargée de mener la réflexion sur la simplification des différentes valeurs, limites, contraintes, recommandations, etc. (une vingtaine) actuellement proposées par la CIPR pour gérer le système de protection radiologique dans différentes situations. La commission principale, lui réclame, pour ses prochaines recommandations, 4 ou 5 valeurs seulement. La tâche est délicate, rude, profitons-en pour lui faire nos suggestions. Le meilleur moyen est d'enrichir de vos remarques et commentaires 
ÉDTTORIAL

le nouveau groupe de réflexion de la SFRP (le troisième depuis le lancement de la réflexion de la CIPR), présidé par Caroline Schieber et Yves Sébastien Cordoliani (ouf ! enfin un homme...). N'oubliez pas non plus de vous exprimer sur ce sujet dans notre lettre à la rédaction.

Henri Métivier

Président du comité de la revue

Vous avez, reçu votre premier numéro (Vol. $38, N^{\circ}$ l) avec retard et nous vous prions de nous en excuser, certaines informations ont pu vous paraître obsolètes. Ce retard lié à des problèmes chez notre imprimeur a été corrigé avec vigueur par EDP Sciences, nous resterons cependant vigilants. 